\title{
CMEARTICLE
}

\section{Clinics in diagnostic imaging (156)}

Hsien Khai $\underline{T a n}^{1}$, MMed, FRCR, Nicholas $\underline{C h e w^{2}}$, MRCP, PhD, Kelvin TL $\underline{C h e w}^{3}$, MBBCh, MSpMed, Wilfred CG $\underline{P e h}^{1}$, FRCP, FRCR

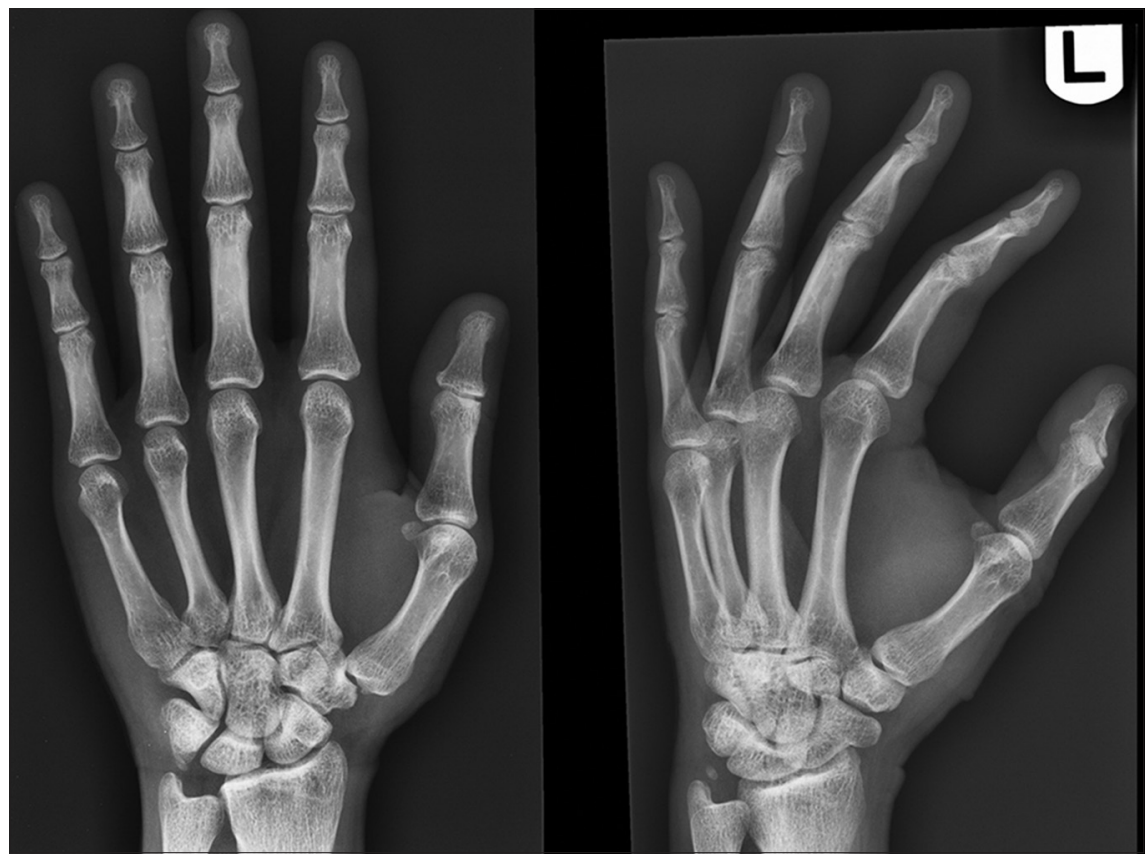

Fig. 1 Frontal (left) and oblique (right) radiographs of the left hand.

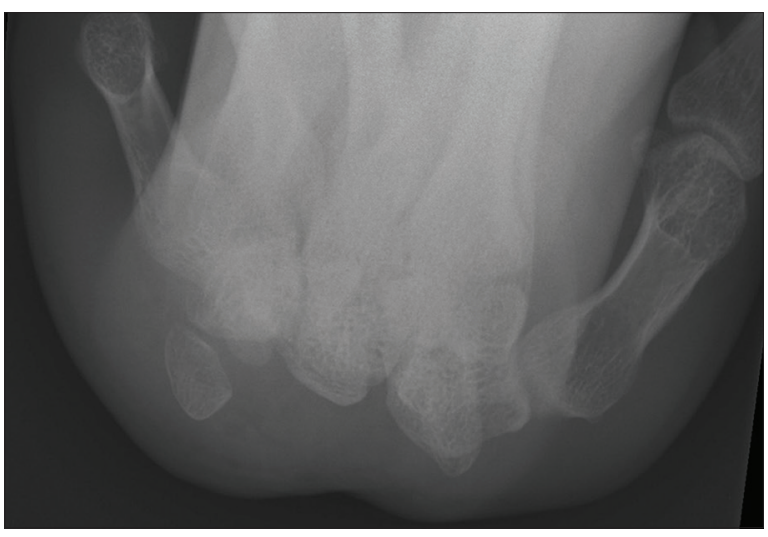

Fig. 2 Carpal tunnel view radiograph of the left wrist.

\section{CLINICAL PRESENTATION}

A 37-year-old, right-handed man with no past medical history presented with pain at the ulnar aspect of his left palm. The pain began after a routine golf practice session at the driving range, during which the patient recalled clipping the grass mat slightly heavily with his golf club while attempting to strike the ball. Subsequently, he experienced mild pain in his left palm while hitting the ball. The patient attributed this to minor soft tissue or ligamentous injury, and continued to practise at the driving range and play on the golf course. The pain gradually worsened over the following four weeks, during which the patient continued

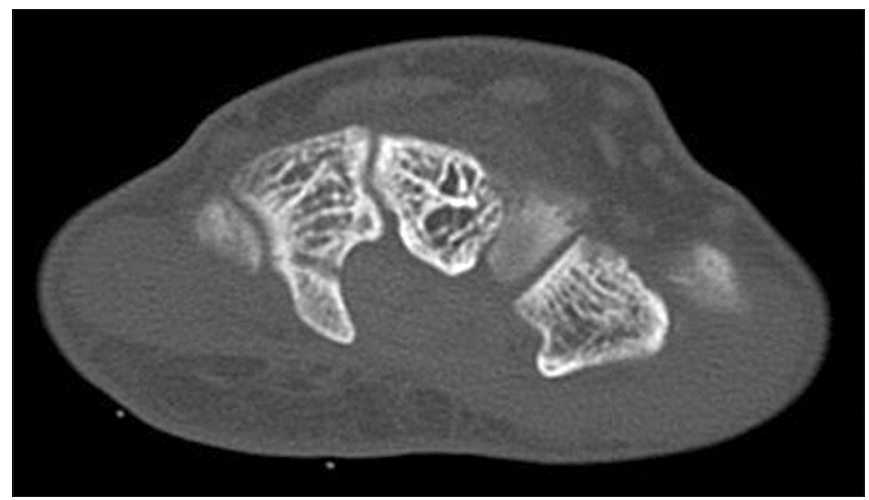

Fig. 3 Axial CT image of the left wrist taken through the level of the mid-hamate bone.

to play golf twice per week. Only when the pain became too severe to hold a golf club or putt a golf ball did he finally seek medical care.

On physical examination, the patient reported mild to moderate pain over the ulnar aspect of the left palm on palpation, the worst over the hypothenar eminence. Pain was exacerbated by ulnar deviation of the affected hand. There was no swelling and no neurological sign or symptom. Radiography of the left hand (Fig. 1) followed by carpal tunnel view radiography (Fig. 2) and computed tomography (CT) of the left wrist (Fig. 3) were performed. What do these images show? What is the diagnosis?

${ }^{1}$ Department of Diagnostic Radiology, Khoo Teck Puat Hospital, 2Division of Infectious Diseases, University Medicine Cluster, National University Health System, ${ }^{3} \mathrm{C} h a n g i$ Sports Medicine Centre, Changi General Hospital, Singapore

Correspondence: Prof Wilfred CG Peh, Senior Consultant and Head, Department of Diagnostic Radiology, Khoo Teck Puat Hospital, Alexandra Health, 90 Yishun Central, Singapore 768828. wilfred.peh@gmail.com. 


\section{IMAGE INTERPRETATION}

Standard radiographs of the left hand and wrist (Fig. 1) did not show any fracture. There was an incidental finding of a well-corticated ossicle adjacent to the ulnar styloid process. As a hook of hamate fracture was suspected from the clinical history and physical examination, carpal tunnel view radiography was performed, but this did not clearly show a hamate fracture due to the overlapping carpal bones (Fig. 2). Axial CT of the left wrist showed a slightly oblique fracture through the base of the hook of the hamate (Fig. 3). There was no fracture displacement.

\section{DIAGNOSIS}

Golf-induced hamate hook fracture.

\section{CLINICAL COURSE}

As the hamate hook fracture was undisplaced and there was no clinical sign of ulnar nerve compromise, the patient was managed conservatively, with cessation of physical activity involving the left hand and wrist for eight weeks. On follow-up at six weeks, significant improvement in the intensity of pain was noted. After eight weeks, the patient resumed playing golf. At the most recent follow-up at two years, the patient had fully recovered and was playing golf without limitation.

\section{DISCUSSION}

Many sporting activities produce specific injuries related to the actions and stresses peculiar to that activity. Knowledge of the sport and its peculiar biomechanics helps the clinician to understand the pathophysiology of injury, hence aiding diagnosis and management. Wrist and hand injuries are commonly encountered in exponents of sports that entail holding an implement to strike a ball, such as golf, baseball and racquet sports. Most of the injuries sustained in golf are not due to a single traumatic incident, but occur as a result of tissue damage occurring over time, typically as a result of repetitive overuse. The majority of golf injuries involving the limbs affect the lead side of the body, i.e. the left side in right-handed golfers. In the upper limb, most injuries occur at impact when the golf club hits the ball or any other object, e.g. the ground or an obscured rock. ${ }^{(1)}$

Among golfers, the commonest injuries affect the lower back, elbow, wrist and hand, followed by the shoulder, knee and other body parts. Professional and recreational golfers, however, sustain different types of injury. In professional golfers, the wrist, back, shoulders and hips are the most commonly injured regions, whereas in recreational golfers, the lower back, elbow and wrist are most frequently injured. ${ }^{(2,3)}$ Among the former, the wrist comprises $20 \%-27 \%$ of all injuries, while among the latter, the incidence is $13 \%-20 \%$. $^{(3-6)}$ In the European Tour, the diagnostic imaging service is a vital part of the structured medical service for professional golfers. Diagnostic and therapeutic ultrasonography is performed on-site, while magnetic resonance (MR) imaging, performed according to predefined protocols, is sent via electronic transfer to supporting musculoskeletal radiologists, who provide immediate reports and liaise with the clinical referrer treating the injured professional golfer. ${ }^{(7)}$
In general, the majority of injuries affecting professional golfers result from overuse, as these high-performance athletes replicate the same swing many thousand times per week, channelling forces through precise musculoskeletal locations and generating clubhead speeds in excess of 100 mph. ${ }^{(2,3)}$ In contrast, the recreational golfer is generally older and less well conditioned in terms of muscular strength, endurance and flexibility. He is likely to play much less frequently, has a poorer swing technique, and is more likely to exacerbate pre-existing conditions or suffer random acute injuries. ${ }^{(6,8-10)}$

Wrist injuries in golf may be classified as a low-impact sports injury. This category of injury typically results in nondisplaced or occult fracture, contusion, stress reaction, ligamentous sprain, tendinopathy and tenosynovitis. This is in contrast to high-impact sports injuries, which produce more severe wrist injuries such as displaced fractures and dislocations, and ligamentous and acute tendon tears. ${ }^{(11)}$ The left wrist is the common site of injury for righthanded golfers, as the golf club is primarily gripped by the left hand, with the handle butt of the club nestled against the hypothenar eminence of the palm. When the clubhead strikes the golf ball or the ground, the wrist and hand absorb most of the impact transmitted along the shaft of the golf club. Repetitive trauma, exacerbated by improper wrist motion, leads to typical wrist injuries affecting golfers, such as hamate hook fracture, ulnar impaction syndrome, de Quervain's disease, and tendinopathies affecting the flexor carpi ulnaris (FCU) and extensor carpi ulnaris (ECU). ${ }^{11}$

Hook of hamate fracture is a well-recognised but uncommon injury seen in golfers. First described in 1972, acute hamate hook fracture may occur when the hamate bone of the leading hand sustains a direct blow from the butt end of the handle of the golf club during the downswing. ${ }^{(12)}$ Stress fracture of the hamate may also result from a sudden change in golf grip or excessive practice. ${ }^{(13)}$ In our patient, there was an initial precipitating acute episode of injury, but the patient's continuation of golf practice probably led to exacerbation of the injury and contributed to the development or worsening of the hamate hook fracture.

The diagnosis of hamate hook fracture is often missed or delayed, as standard radiographs of the wrist and hand almost never demonstrate the fracture. A special projection, the carpal tunnel view radiograph, has been advocated in the past for detection of this fracture. However, full wrist extension, which is required for this projection, may be difficult to achieve, as maintaining this position may be painful for the patient. In many centres, the carpal tunnel view has been replaced by CT. ${ }^{(14)} \mathrm{CT}$ has an accuracy of $97 \%-100 \%$ for the detection of this fracture type. ${ }^{(15)}$ In our patient, both the standard and carpal tunnel view radiographs failed to show the fracture, and the diagnosis was made on CT. MR imaging can also be used to show fractures of the hamate hook, although this diagnosis may be difficult if the fracture is chronic and there is no bone marrow oedema.

After a diagnosis of hamate hook fracture is confirmed, a trial of casting for six weeks is indicated if symptoms can be relieved by simple immobilisation. However, if the patient remains symptomatic after $6-8$ weeks of cast immobilisation, operative excision of the hamate hook fracture fragment may be needed to 

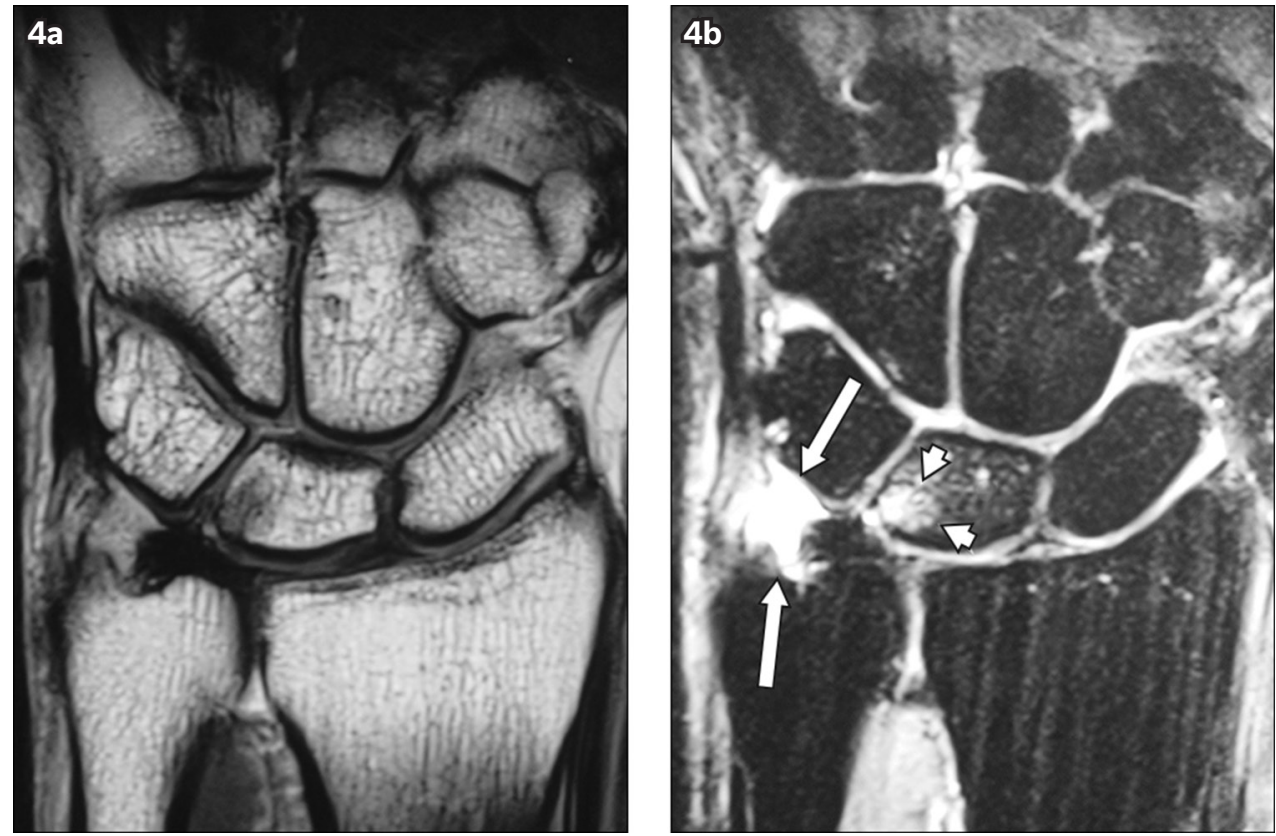

Fig. 4 Ulnar impaction in a 51-year-old, right-handed male golfer. Coronal (a) fast spin-echo proton densityweighted and (b) STIR MR images show slight positive ulnar variance and patchy marrow oedema in the ulnar aspect of the lunate (arrowheads), with overlying cartilage thinning and irregularity of the ulnar-sided proximal surface of the lunate. There is fluid-filled separation of the ulnar attachment of the TFCC from the ulnar styloid process (arrows).
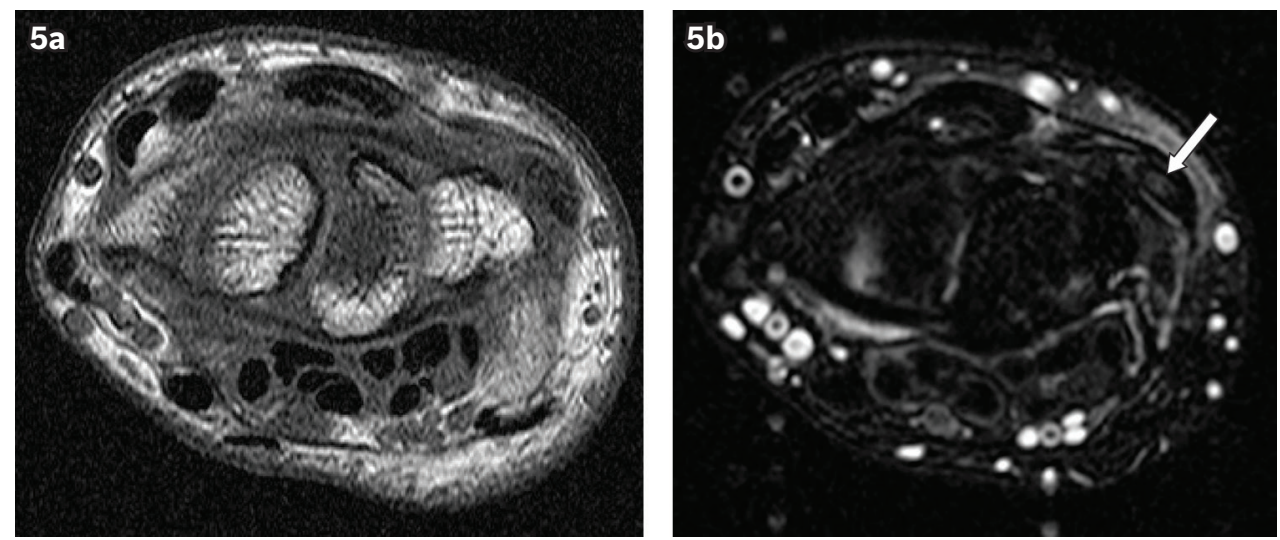

Fig. 5 Extensor carpi ulnaris (ECU) tendinosis in a 40-year-old right-handed male golfer. Axial (a) T1-W and (b) T2-W MR images of the wrist, taken at the level of the proximal carpal row, show slight thickening and T2-hyperintense signal (arrow) within the otherwise intact ECU tendon, diagnostic of tendinosis.

relieve pain or improve grip strength. ${ }^{(16,17)}$ Internal fixation using both Kirschner wires and screw fixation, with good outcomes, has been reported, although this procedure may be technically difficult. ${ }^{(17)}$ Our patient responded very well to conservative measures and did not require any operative intervention.

Golfers may develop ulnar impaction syndrome, also known as ulnar abutment or ulnocarpal loading. This is a degenerative condition caused by the ulnar head impacting upon the ulnar-sided carpus, with injury to the triangular fibrocartilage complex (TFCC). The continuum of pathological changes includes TFCC degenerative tear; chondromalacia of the lunate, triquetral and distal ulnar head; lunotriquetral ligament tear; and osteoarthritis of the ulnocarpal and distal radioulnar joints. ${ }^{(18)}$ Any condition that results in a relative increase in ulnar variance, such as firm grip, pronation and ulnar deviation of the wrist, can exacerbate symptoms. A majority of ulnar impactions is associated with positive ulnar variance or increased dorsal tilt of the distal radius. While the injury is rare in the absence of anatomical predispositions, it can occur if there is excessive repeated loading of the ulnar-sided carpus. ${ }^{(19)} \mathrm{MR}$ imaging is useful in demonstrating the various stages of ulnar impaction syndrome, such as radiographically occult bone marrow oedema of the lunate, and TFCC and lunotriquetral ligament tears (Fig. 4). ${ }^{(18)}$

In golf, tendinopathy results from a sudden increase in practice intensity, or grip alteration that causes increased loading in an unaccustomed part of the wrist. ${ }^{(19,20)}$ The FCU tendon of the wrist in right-handed golfers may be injured due to microtrauma from forces produced by the swing just prior to impact. When the club hits the ground or takes a divot, there is sudden overload of the flexor tendon, leading to injury. ${ }^{(19)}$ The ECU tendon may be injured when the golfer repeatedly uncocks his wrists in the early part of the downswing, leading to repetitive overloading, microtrauma and injury to this tendon (Fig. 5). ${ }^{(19)}$ 

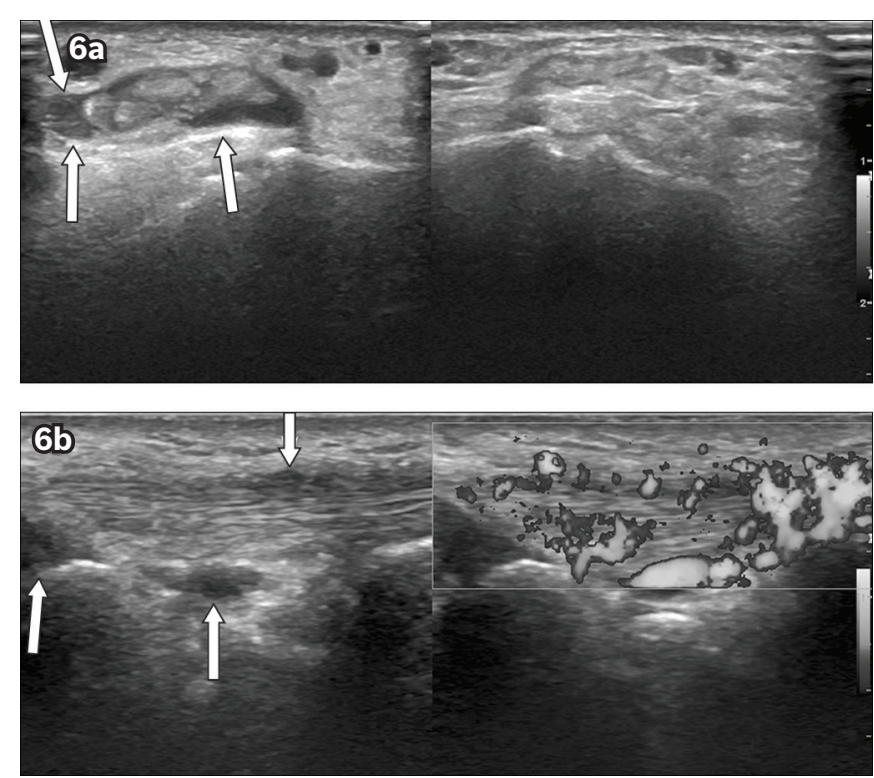

Fig. 6 De Quervain's tenosynovitis in a 61-year-old female non-golfer (a) Transverse US image taken through the snuff box of the right wrist shows an effusion (arrows) within the tendon sheath of the right abductor pollicis longus (APL) tendon (left), compared to the normal tendon in the left snuff box (right). (b) Longitudinal US image of the same tendon shows effusion (arrows) surrounding the APL tendon (left) and increased colour Doppler signal within the thickened tendon sheath, indicating inflammation (right).

De Quervain's disease refers to tenosynovitis or tendinosis of the abductor pollicis longus (APL) and extensor pollicis brevis (EPB) tendons of the thumb. It is a type of overuse injury that typically occurs in the left wrist of right-handed golfers at the top of their backswing, particularly when there is a tight grip of the club and repeated ulnar deviation during the golf swing. (2)

Ultrasonography and MR imaging are useful for confirming the diagnosis of tendinopathy and to help differentiate tendinopathy from other conditions in situations where the clinical presentation is equivocal. MR imaging features of tenosynovitis include T2 hyperintense fluid within a thickened and distended tendon sheath, while appearances of tendinosis can range from mild tendon swelling with increased T2 signal change (Fig. 5) to longitudinal splitting of the tendon in severe cases. Ultrasonography is comparable to MR imaging for diagnosing tenosynovitis and tendinosis, but is highly operator-dependent (Fig. 6). Other less common golfing injuries include trigger finger, carpal tunnel syndrome, median nerve compression neuropathy, ECU tendon dislocation and ulnar artery thrombosis. ${ }^{(1)}$

In summary, a variety of specific injury patterns may afflict golfers, and are related to unique actions and stresses. Knowledge of the sport and how it is played helps in diagnosis and management of injury patterns, while knowledge of the biomechanics behind the golf swing is useful for understanding the pathophysiology of wrist injuries. Imaging plays an important role in the evaluation and diagnosis of the range of bone and soft tissue injuries in golf.
ABSTRACT The wrist is a common site of injury and the most frequently injured body part among professional golfers. A 37-year-old, right-handed male golfer presented with pain at the ulnar aspect of his left palm, which grew worse after an initial traumatic impact from the golf club handle. There was tenderness over the hypothenar eminence of the left palm. Computed tomography of the left wrist showed an undisplaced fracture through the base of the hamate hook. The golf-induced hamate hook fracture was managed conservatively, with cessation of physical activity involving the left hand and wrist for eight weeks. The patient made a full recovery. Repetitive trauma, exacerbated by improper wrist motion, leads to typical wrist injuries affecting golfers, such as ulnar impaction syndrome, de Quervain's disease, and tendinopathy affecting the flexor carpi ulnaris and extensor carpi ulnaris, all of which can be diagnosed on imaging.

Keywords: golf injury, hamate fracture, tendinopathy, ulnar impaction syndrome, wrist injury

\section{REFERENCES}

1. McHardy AJ, Pollard HP. Golf and upper limb injuries: a summary and review of the literature. Chiropr Osteopat 2005; 13:7.

2. McCarroll JR. Overuse injuries of the upper extremity in golf. Clin Sports Med 2001; 20:469-79.

3. Gosheger G, Liem D, Ludwig K, Greshake O, Winkelmann W. Injuries and overuse syndromes in golf. Am J Sports Med 2003; 31:438-43.

4. McCarroll JR, Gioe TJ. Professional golfers and the price they pay. Phys Sports Med 1982; 10:64-70.

5. McCarroll JR, Rettig AC, Shelbourne KD. Injuries in the amateur golfer. Phys Sports Med 1990; 18:122-6.

6. Batt ME. A survey of golf injuries in amateur golfers. Br J Sports Med 1992; 26:63-5.

7. O'Connor PJ, Hawkes R. Imaging the elite golfer. Skeletal Radiol 2013; 42:607-9.

8. Kim DH, Millett PJ, Warner JJ, Jobe FW. Shoulder injuries in golf. Am J Sports Med 2004; 32:1324-30.

9. Wiesler ER, Lumsden B. Golf injuries of the upper extremity. J Surg Orthop Adv 2005; 14:1-7.

10. Bayes MC, Wadsworth LT. Upper extremity injuries in golf. Phys Sportsmed 2009; 37:92-6.

11. Bancroft LW. Wrist injuries: a comparison between high- and low-impact sports. Radiol Clin North Am 2013; 51:299-311.

12. Torisu T. Fracture of the hook of the hamate by a golfswing. Clin Orthop Relat Res 1972; 83:91-4.

13. Guha AR, Marynissen $\mathrm{H}$. Stress fracture of the hook of the hamate. Br J Sports Med 2002; 36:224-5.

14. Rubin DA, Daffner RH, Weissman BN, et al. Expert Panel on Musculoskeletal Imaging. ACR Appropriateness Criteria ${ }^{\circledR}$ acute hand and wrist trauma. In: Reston (VA): American College of Radiology (ACR) 2008 [online]. Available at: http://www.acr.org/ /media/ODA5C508B7E14323A1AE52F23543F093. pdf. Accessed November 1, 2013

15. Andresen R, Radmer S, Sparmann M, Bogusch G, Banzer D. Imaging of hamate bone fractures in conventional X-rays and high-resolution computed tomography. An in vitro study. Invest Radiol 1999; 34:46-50.

16. O'Grady W, Hazle C. Persistent wrist pain in a mature golfer. Int J Sports Phys Ther 2012; 7:425-32.

17. Scheufler O, Andresen R, Radmer S, et al. Hook of hamate fractures: critical evaluation of different therapeutic procedures. Plast Reconstr Surg 2005; 115:488-97.

18. Cerezal L, del Piñal F, Abascal F, et al. Imaging findings in ulnar-sided wrist impaction syndromes. Radiographics 2002; 22:105-21.

19. Murray PM, Cooney WP. Golf-induced injuries of the wrist. Clin Sports Med 1996; 15:85-109.

20. Clancy WG Jr, Hagan SV. Tendinitis in golf. Clin Sports Med 1996; 15:27-35. 


\section{SINGAPORE MEDICAL COUNCIL CATEGORY 3B CME PROGRAMME} (Code SMJ 201410B)

Question 1. Regarding hook of hamate fractures:

(a) This fracture may result from a direct blow from the handle of a golf club.

(b) Stress fractures may occur.

(c) Diagnosis is often missed or delayed on standard radiographs due to overlapping bones.

(d) Computed tomography has a $5 \%-10 \%$ accuracy for detection of hamate fractures.

Question 2. In ulnar impaction syndrome:

(a) The ulnar head impacts the ulnar-sided carpus.

(b) The triangular fibrocartilage complex is spared.

(c) It is associated with negative ulnar variance.

(d) Magnetic resonance (MR) imaging is useful for demonstrating the various stages of injury.

Question 3. De Quervain's disease:

(a) Refers to tenosynovitis of the abductor pollicis brevis and extensor pollicis longus.

(b) Typically involves the tendons of the index finger.

(c) Occurs due to repeated radial deviation during the golf swing.

(d) Can be diagnosed on ultrasonography and MR imaging.

Question 4. In imaging of tendinopathies:

(a) Tenosynovitis appears as a thickened and distended tendon sheath with T2-hyperintense fluid on MR imaging.

(b) Tendinosis ranges from tendon swelling with increased T2 signal to longitudinal splitting of the tendon on MR imaging.

(c) Ultrasonography is less sensitive than MR imaging as a diagnostic tool.

(d) Ultrasonography is not operator-dependent.

Question 5. Regarding wrist injuries in golfers:

(a) They are mostly due to a single traumatic event.

(b) Most injuries are left-sided in a right-handed golfer.

(c) The incidence of wrist injuries is lower in professional golfers than recreational golfers due to better swing technique.

(d) Wrist injuries are classified as a high-impact injury.

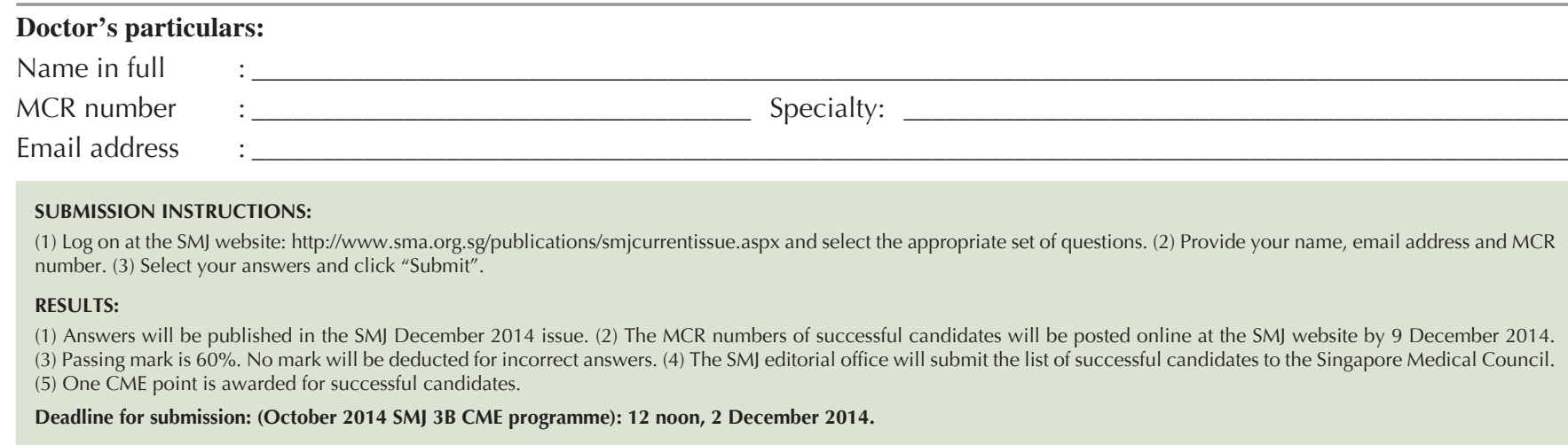

